\title{
EUV Observation with Normal Incidence Multilayer Telescopes
}

\author{
HIDEYO KUNIEDA, ${ }^{1}$ KOUJUN YAMASHITA, ${ }^{1}$ \\ TAKASHI YAMAZAKI, ${ }^{1}$ KAZUAKI IKEDA, ${ }^{1}$ \\ KAZUTAMI MISAKI, ${ }^{1}$ YOSHIYUKI TAKIZAWA, ${ }^{2}$ \\ MASATO NAKAMURA, ${ }^{2}$ ICHIRO YOSHIKAWA, \\ AND ASAMI YAMAGUCHI ${ }^{3}$ \\ ${ }^{1}$ Department of Physics, Nagoya University, Nagoya 464-01 \\ ${ }^{2}$ Department of Earth and Planetary Physics, University of Tokyo, Tokyo 113 \\ ${ }^{3}$ National Astronomical Observatory, Mitaka 181
}

The EUV emission from hot interstellar plasmas is observed by normal incidence telescopes on board a sounding rocket. It was performed on January 29,1995 , to observe the sky area around the HZ 43 close to the north galactic pole. The wave bands $(\delta \lambda \sim 10 \AA)$ are provided at 130 and $170 \AA$ by the multilayer coating on the reflectors of $20 \mathrm{~cm}$ in diameter and of $30 \mathrm{~cm}$ in focal length. The focal plane images are detected by CsI coated MCP's. The observed flux of HZ 43 is 1.5 counts $\mathrm{s}^{-1}$ at $130 \AA$ and 3 counts $\mathrm{s}^{-1}$ at $170 \AA$. The diffuse emission is $27 \mathrm{c} / \mathrm{s} / \mathrm{deg}^{2}$ at $130 \AA$ and $20 \mathrm{c} / \mathrm{s} / \mathrm{deg}^{2}$ at $170 \AA$. Those preliminary numbers are subjects to change along with the data analysis.

\section{Introduction}

Interstellar field is assumed to be filled with cold gas with dust and thin hot plasmas of about $10^{5}$ to $10^{6} \mathrm{~K}$. The latter component has been recognized in soft X-ray observations below $1 \mathrm{keV}$. The plasmas of $10^{6} \mathrm{~K}$ is characterized by emission lines from $\mathrm{O}$ VII and OVIII at 0.56 and $0.65 \mathrm{keV}$, respectively, while EUV emission lines are expected from plasmas of lower temperature in $10^{5} \mathrm{~K}$ range.

Thinner windows were developed to enhance the detection efficiency in longer wavelength range for the proportional counters. However, strong contamination was found due to the He II (304 $\AA)$ emission lines from geocorona. The diffuse EUV emission is difficult to separate from such strong geocorona emission, because spectrometer is not available but filtering, which allows only factor of ten reduction of He II lines even around the absorption edges.

A breakthrough comes from the progress of X-ray optics. Multilayer coatings, thin layer pairs of light and heavy elements, constructed on mirror surfaces efficiently reflect $\mathrm{X}$-rays of selected wavelength. The first mission to measure celestial objects is performed with $A L E X I S$ (see the paper in the same issue). In order to improve sensitivity, large normal incidence telescopes are prepared for a Japanese rocket mission in two wave bands at 130 and $170 \AA$ to observe the sky region around the brightest EUV source HZ 43 . The flight was successful and some complex structure of EUV emission is observed together with a bright point source, $\mathrm{HZ} 43$.

\section{Emission Lines from Hot Interstellar Medium}

Kato (1976) calculated radiation of a hot thin plasmas from 1 to $250 \AA$. The lower temperature plasmas than $10^{6} \mathrm{~K}$ are expected to emit EUV lines. Figure 1 shows the line emission power in various wavelength bands as a function of temperature (quoted 


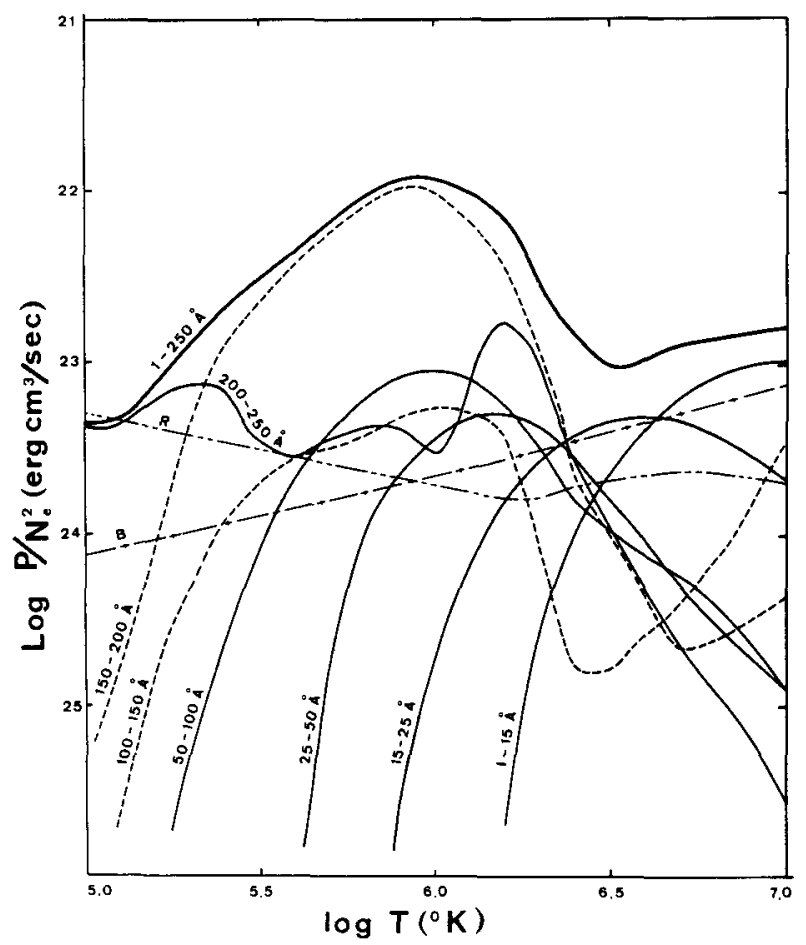

FIgURE 1. Line emission power vs. $T$.

from Fig. 4 of Kato 1976). The plasmas below $10^{6} \mathrm{~K}$ are characterized by strong lines in 150-200 $\AA$. Covering 10-100 $\AA$, very soft X-ray detectors only detect the small portion of energy flux from cooler gas. The calculated spectrum of $8 \times 10^{5} \mathrm{~K}$ is shown in Fig. 2 in wave length range from 100 through $250 \AA$ (Fig. 3 of Kato 1976). The strongest lines are found at around $170 \AA$, all emerged from ionized iron ions (Fe IX 171.06 $\AA, \mathrm{Fe} X$ $170.9 \AA$ ). Below $140 \AA$, no lines are seen more than $1 / 100$ of the strongest one at $170 \AA$.

Our strategy is to provide one pass band at $170 \AA$ to get those strong lines and another band at $130 \AA$ to put a limit of continuum flux without line emission. The lines at 170 $\AA$ have strong temperature dependence, which is clear in the Fig. 1.

\section{Instrumentation}

\subsection{Multilayer Normal Incidence Telescopes}

Since the two wave bands at 130 and $170 \AA$ are favorable to decide the physical situation of hot plasmas at the temperature of $10^{5}-10^{6} \mathrm{~K}, 2 \mathrm{~d}$ of multilayer coatings are tuned to be $140 \AA$ and $184 \AA$ with 20 and 25 layer pairs of Mo/Si, respectively. The reflectivity measured at a synchrotron facility (UVSOR at the Institute for Molecular Science: Okazaki, Japan) are shown in Fig. $3 \mathrm{a}$ and $3 \mathrm{~b}$. Peak reflectivity is about $50-60 \%$ and the width of pass band is about $10 \AA$ in both cases.

Multilayer structure is coated on a spherical mirror of $20 \mathrm{~cm}$ in diameter and $30 \mathrm{~cm}$ in focal length. Polishing and coatings were done by Nikon. It is placed at the bottom 

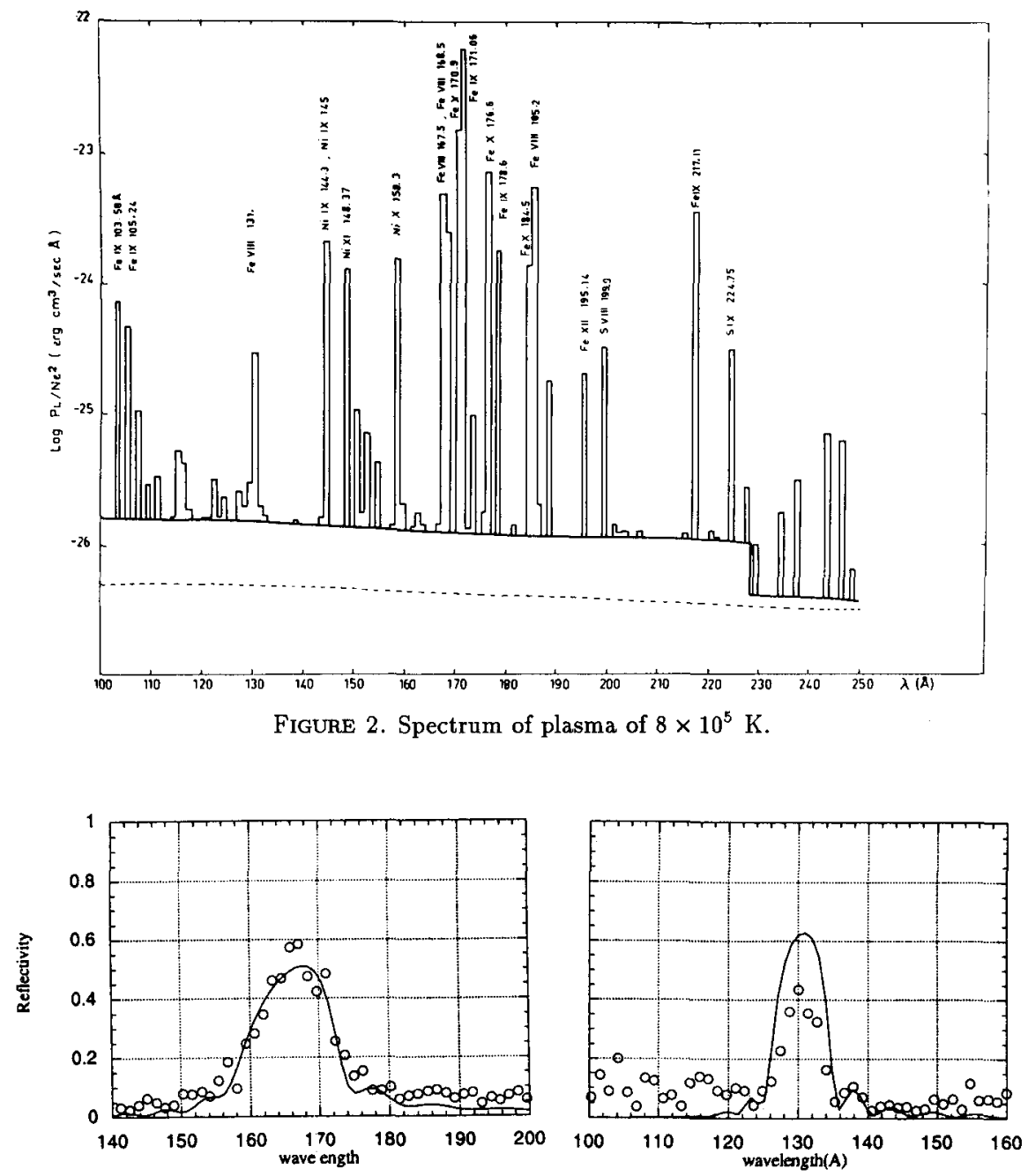

Figure 3. (a) Mirror reflectivity (NIT-1). (b) Mirror reflectivity (NIT-2).

of a housing, whose cross section is shown in Fig. 4. A micro-channel plate of $28 \mathrm{~mm}$ in diameter (corresponding field of view of 4 degree) is put on the focal plane. Images are obtained by a registive plate after the two stage MCP. The CsI coating on the MCP yields detection efficiency of about $20 \%$ at $130-170 \AA$. In order to reject longer wave light $\mathrm{C}$ filter of $1200 \AA$ is placed in front of MCP. The inflow of the ambient plasmas is rejected by a mesh at the entrance window. The normal incidence telescope for $170 \AA$ is named NIT-1 and that for $130 \AA$ is named NIT-2.

\subsection{Helium Line Monitor and UV Star Sensor}

Another multilayer telescope(HEM) is equipped to monitor He II lines(304 $\AA$ ) from geocorona. One more telescope (UVT) with mono-layer coating is prepared as a attitude 


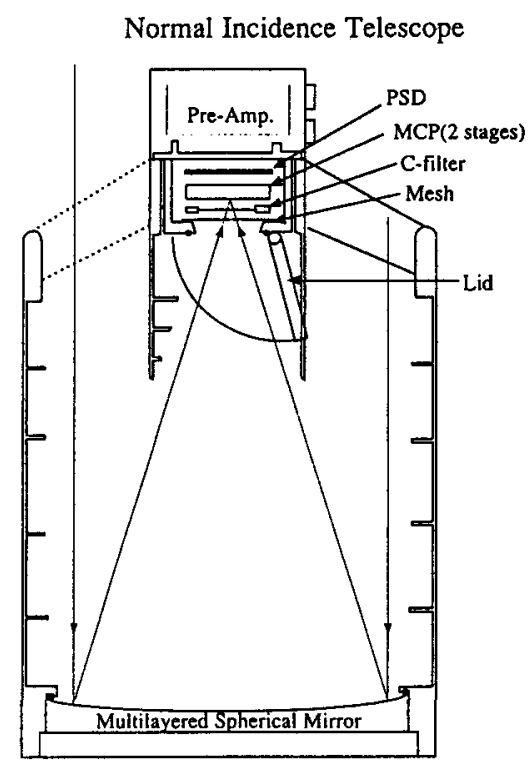

FIGURE 4. Cross section of NIT.

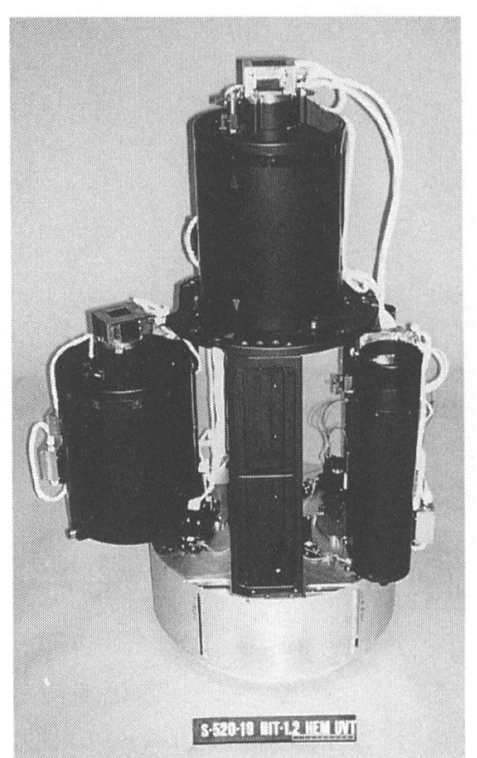

Figure 5. Picture of Hole Payload.

sensor with stars in UV band $(1600-2800 \AA)$. Four photomultiplyers are placed on the focal plane with rotating slits of three different patterns.

NIT-1 is placed at the top of the structure, while NIT-2, UVT and HEM are flooded inside the structure, which are to be deployed after the nose fairing is removed in the sky (Fig. 5). All these instruments are looking at the top direction of the structure.

\section{Flight Operation}

The telescopes were launched by a rocket S-520-19 at 16:00 (UT) on 1995 January 28 from Kagoshima Space Center of the Institute of Space and Astronautical Science. $40 \mathrm{~s}$ after the launch, nose fairing was blown off and the attitude control system, deploy of instruments, and high voltage operations were taken place in sequence. At $X+100$ sec in the altitude of more than $150 \mathrm{~km}$, all instruments were ready to observe the sky area around HZ 43. During 300 s pointing observation, payload achieved at the apogee of 350 $\mathrm{km}$. Then the system was rotated 360 degree around the pointing direction for $50 \mathrm{sec}$. Telemetry data was successfully obtained by the end of our observation, with some short interruptions.

\section{Results}

\subsection{Light Curve}

Total counting rate of whole detector is examined for each detector after the start of operation at $100 \mathrm{~s}$. It is almost constant at the level of 300 counts $\mathrm{s}^{-1}$ and 500 counts $\mathrm{s}^{-1}$ for NIT-1 and NIT-2, respectively, except for the three occasions at $130 \mathrm{~s}, 180$ sec, and $400 \mathrm{~s}$, when the telemetry was lost. Those counting rates could have altitude dependence, if there were some geophysical contaminations due to the air glow and the 


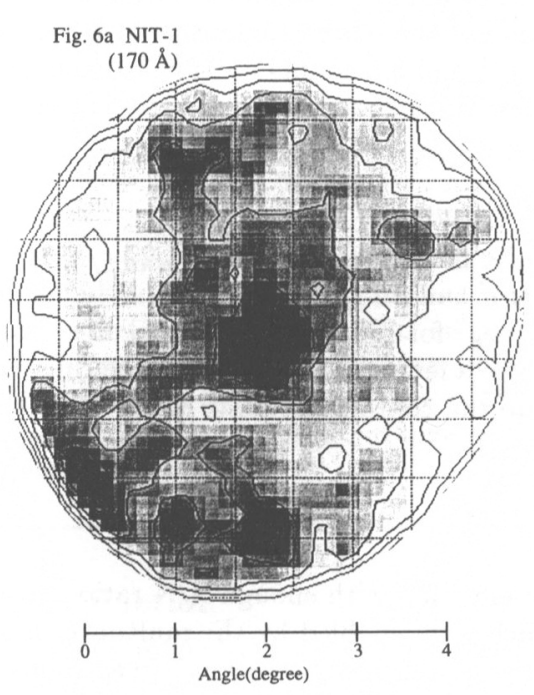

Figure 6. (a) NIT-1, $170 \AA$.

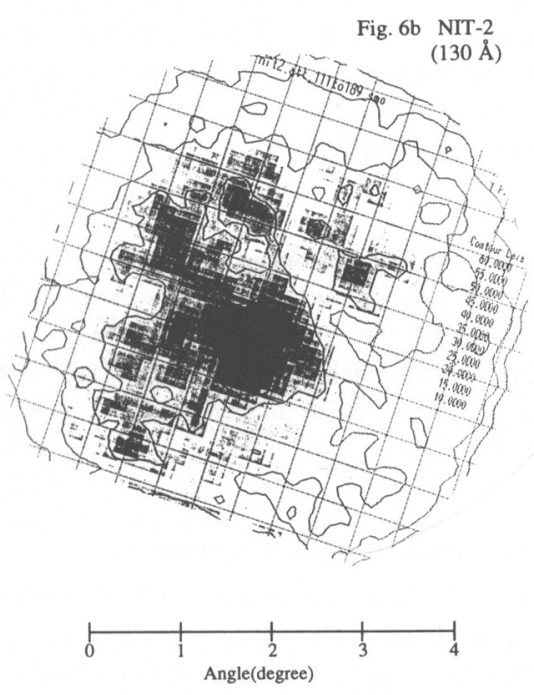

(b) NIT-2, $130 \AA$.

radiation belts. Electrons trapped by geomagnetic field should be sensitive to the angle between the line of sight and the magnetic field. We are able to put an upper limit of about $10 \%$ for the contamination from geophysical origins.

\subsection{Images}

Fig. $6 \mathrm{a}$ shows the NIT- 1 image of $170 \AA$ band integrated from $\mathrm{X}+200$ through $\mathrm{X}+$ $350 \mathrm{~s}$ without attitude correction. The bright source at the center is the image of $\mathrm{HZ} 43$. The image size of a point source is estimated to be 0.3 degree or so in this case. Other structure found right top, right bottom and a sort of "peninsula" to the left could be real feature.

Fig. $6 \mathrm{~b}$ is the NIT-2 image of $130 \AA$ band in the same period of time. The bright source is not so much out standing as in Fig. 6a, but there. Other structures seen in 170 $\AA$ image are also found.

\section{Discussion}

\subsection{Detection Efficiency}

The effective area and detection efficiency are roughly estimated as follows.

\begin{tabular}{lllllll}
\hline Eff. Area & Ref. & $d \lambda$ & Detector & Filter & Mesh \\
$\left(300 \mathrm{~cm}^{2}\right) \times$ & $(50 \%) \times$ & $(5 \AA) \times$ & $(20 \%) \times$ & $(30 \%) \times$ & $(90 \%)$ & $=41 \mathrm{~cm}^{2} \AA(130 \AA)$ \\
$\left(300 \mathrm{~cm}^{2}\right) \times$ & $(50 \%) \times$ & $(10 \AA) \times$ & $(20 \%) \times$ & $(20 \%) \times$ & $(90 \%)$ & $=54 \mathrm{~cm}^{2} \AA(170 \AA)$ \\
\hline
\end{tabular}




\subsection{Intensity of $\mathrm{HZ} 43$}

The flux of $\mathrm{HZ} 43$ in both bands is estimated to be 3 and 1.5 counts $\mathrm{s}^{-1}$ from the central enhancement from the diffuse emission. If one use the observe intensity of $\mathrm{HZ} 43$ with EUVE, estimated counting rate is 7.6 counts $\mathrm{s}^{-1}$ and 3.5 counts $\mathrm{s}^{-1}$ for 170 and $130 \AA$, respectively. Though the absolute value is a few times lower, the flux ratio is consistent with the reported value.

\subsection{Diffuse EUV Emission}

The observed diffuse emission averaged over the field of view is 20 and $27 \mathrm{c} / \mathrm{s} / \mathrm{deg}^{2}$ for 170 and $130 \AA$. Using the effective area of $5.4 \mathrm{~cm}^{2}$ and $8.2 \mathrm{~cm}^{2}$ for a line, the flux of Fe IX at $170 \AA$ is $3.7 \mathrm{c} / \mathrm{s} / \mathrm{cm}^{2} / \mathrm{deg}^{2}$ and $3.3 \mathrm{c} / \mathrm{s} / \mathrm{cm}^{2} / \mathrm{deg}^{2}$ for $130 \AA$. If the normal abundance is assumed, the temerature of gas is suggested to be a few time $10^{5}$ based on the calculation in Fig. 1. However, it is necessary to evaluate the contamination to those wave bands very carefully.

\section{Conclusion and Summary}

This is the first observation of EUV diffuse emission with enough $\mathrm{S} / \mathrm{N}$ ratio and with good spatial resolution of quarter degree, which were enabled by the multilayer normal incidence telescopes. It confirms the possibilities of multilayer telescopes in astrophysical observations. The data analysis is still going on and the results presented here are subjects to change.

\section{REFERENCES}

Kato, T. 1976, Radiation from Hot Thin Plasmas from 1 to $250 \AA$, ApJS, 30, 397 